\section{Growth hormone plus resistance exercise attenuate structural changes in rat myotendinous junctions resulting from chronic unloading}

D. Curzi, ${ }^{1}$ D. Lattanzi, ${ }^{1}$ S. Ciuffoli, ${ }^{1}$

S. Burattini, ${ }^{1}$ R.E. Grindeland, ${ }^{2}$

V.R. Edgerton, ${ }^{3,4}$ R.R. Roy, ${ }^{3}$ J.G. Tidball, ${ }^{5}$

E. Falcieri ${ }^{1,6}$

'Department of Earth, Life and

Environmental Sciences, University of

Urbino Carlo Bo, Italy

${ }^{2}$ Life Sciences Division, NASA - Ames

Research Center, Moffett Field, CA, USA

${ }^{3}$ Department of Integrative Biology and

Physiology and the Brain Research Inst.,

University of California, Los Angeles, CA,

USA

${ }^{4}$ Department of Neurology and

Neurosurgery, University of California,

Los Angeles, CA, USA

${ }^{5}$ Molecular, Cellular \& Integrative

Physiology Program, University of

California, Los Angeles, CA, USA

${ }^{6} \mathrm{CNR}$, Molecular Genetics Institute and

Rizzoli Orthopaedic Institute, Bologna,

Italy

\section{Abstract}

Myotendinous junctions (MTJs) are specialized sites on the muscle surface where forces generated by myofibrils are transmitted across the sarcolemma to the extracellular matrix. At the ultrastructural level, the interface between the sarcolemma and extracellular matrix is highly folded and interdigitated at these junctions. In this study, the effect of exercise and growth hormone $(\mathrm{GH})$ treatments on the changes in MTJ structure that occur during muscle unloading, has been analyzed. Twenty hypophysectomized rats were assigned randomly to one of five groups: ambulatory control, hindlimb unloaded, hindlimb unloaded plus exercise ( 3 daily bouts of 10 climbs up a ladder with $50 \%$ body wt attached to the tail), hindlimb unloaded plus GH (2 daily injections of $1 \mathrm{mg} / \mathrm{kg}$ body wt, i.p.), and hindlimb unloaded plus exercise plus GH. MTJs of the plantaris muscle were analyzed by electron microscopy and the contact between muscle and tendon was evaluated using an IL/B ratio, where $B$ is the base and $I L$ is the interface length of MTJ's digit-like processes. After 10 days of unloading, the mean IL/B ratio was significantly lower in unloaded (3.92), unloaded plus exercise (4.18), and unloaded plus $\mathrm{GH}$ (5.25) groups than in the ambulatory control (6.39) group. On the opposite, the mean IL/B ratio in the group treated with both exercise and GH (7.3) was similar to control. These findings indicate that the interaction between exercise and $\mathrm{GH}$ treatments attenuates the changes in MTJ structure that result from chronic unloading and thus can be used as a countermeasure to these adaptations.

\section{Introduction}

Myotendinous junctions (MTJs) are sites where force generated by muscle is transmitted across the muscle cell membrane to the extracellular matrix (ECM). At these sites, the terminal sarcomeres extend into digit-like extensions of the cell into the surrounding connective tissue. The extensively folded MTJ membrane amplifies the muscle-tendon interface, so that contact area between muscle and tendon is increased by a factor of 10 to $50 .^{1}$

MTJ structure is modified following muscle denervation, modified muscle rest length or modified loading. Four-weeks of denervation of frog semitendinosus muscles produced disruptions in the structure of the basement membrane at the MTJ, caused a reduction of subsarcolemmal densities and an increased concentration of vesicles in the junctional processes, ${ }^{2}$ while stretching muscle to a new rest length led to the accumulation of polysomes and mitochondria, the appearance of nascent sarcomeres, and elevated concentrations of mRNA for myosin heavy chain at the MTJ. ${ }^{3}$ Reductions in muscle loading associated with spaceflight also produced a substantial decrease in the muscle-tendon interface at the MTJs of rats, as well as an increase in ribosome and mitochondria number. ${ }^{4,5}$ Similarly, human muscle wasting that followed lower leg amputation was accompanied by reduced contact between muscle and tendon at MTJs. ${ }^{6}$ Aging rat muscles also showed changes in MTJs that included extensive collagen deposition adjacent to this region, alterations in the length and the shape of the finger-like processes and thickening of sarcoplasmic invaginations. ${ }^{7}$ However, increased muscle use can also cause changes in this structure. A moderate treadmill-running regimen applied to rats increased the percentage of the branched tendon interdigitations at the MTJ and the number of bifurcations of the digit-like processes. $^{8}$

These modifications in muscle structure and protein synthesis that are restricted to MTJs in muscle undergoing modified loading suggest that these aspects of muscle remodeling are locally regulated in the muscle fiber.
Correspondence: Prof. Elisabetta Falcieri, DiSTeVA, Urbino University Carlo Bo, via Ca' le Suore, 61029 Urbino (PU), Italy.

Tel. +39.0722.304284 - Fax: +39.0722 .304244 .

E-mail: elisabetta.falcieri@uniurb.it

Key words: Myotendinous junction, ultrastructure, exercise, growth hormone, atrophy, disuse, unloading.

JGT and EF contributed equally to the manuscript.

Acknowledgments: the authors are indebted to Lorenzo Bedini, Oliviero Rusciadelli, Federico Bastianelli, and Aurelio Valmori for skillful technical assistance. The research was supported by Urbino University and the Ministry of Education, University and Research (PRIN 2009).

Received for publication: 10 September 2013. Accepted for publication: 4 October 2013.

This work is licensed under a Creative Commons Attribution NonCommercial 3.0 License (CC BYNC 3.0).

(C)Copyright D. Curzi et al., 2013

Licensee PAGEPress, Italy

European Journal of Histochemistry 2013; 57:e37 doi:10.4081/ejh.2013.e37

Although loss of MTJ structural complexity is associated with loss of MTJ strength that may be functionally important, ${ }^{9}$ interventions that are sufficient to obviate losses of MTJ structural complexity and strength are unexplored. Changes in muscle loading can also produce large and rapid changes in muscle mass, which suggests the possibility that factors that regulate muscle mass changes could also regulate MTJ structural complexity. Rapid changes in muscle mass occur in numerous conditions including chronic diseases, aging, limb immobilization, or prolonged bed rest due to injury or trauma. ${ }^{10-12}$ In at least some of these conditions of muscle mass loss, the endocrine influences mediated by growth hormone $(\mathrm{GH})$ and insulin-like growth factor 1 (IGF-1) can slow or prevent muscle mass loss. ${ }^{13} \mathrm{GH}$ can elevate the expression of IGF-1 that then functions a potent mitogen that can induce proliferation of satellite cells ${ }^{14}$ and shift muscle to a positive protein balance during muscle hypertrophy. ${ }^{15}$ These anabolic influences of IGF1 can be signaling through two different intracellular signal transduction pathways that regulate muscle proliferation or muscle differentiation. ${ }^{16,17}$ However, whether signaling via GH/IGF1 can also modulate MTJ structural complexity during modified muscle use is unknown.

Increased muscle use can also protect muscle mass during muscle wasting conditions that are associated with stroke, sepsis, cancer, and AIDS. Exercise training alone or combined 
with nutritional supplementation has been shown to induce skeletal muscle hypertrophy, thus aiding rehabilitation or reducing muscle wasting. ${ }^{18,19}$ During rehabilitation, exerciseinduced myogenesis may be in part responsible for the recovery of muscle mass. ${ }^{20}$ Several interventions aimed to slow or prevent muscle mass loss during periods of disuse have been explored, indicating that either intermittent muscle training or pharmacological treatments could slow atrophy. For example, a combination of daily resistance exercise and $\mathrm{GH}$ treatment was capable of maintaining nearly all of the myofibrillar protein content, increasing protein synthesis in the gastrocnemius of hind-limb unloaded rats. ${ }^{21}$

In the current investigation, we test the hypothesis that loss of MTJ structural complexity that occurs during reduced muscle loading can be attenuated by $\mathrm{GH}$ treatments or intermittent exercise. We also propose that combined administration of $\mathrm{GH}$ with intermittent exercise have an additive effect on protecting MTJ structure. Finally, we compare the protective effects of $\mathrm{GH}$ treatments and exercise on MTJ structure with their influences on muscle mass, to assess whether similar regulatory processes are involved in maintaining MTJ structure and muscle mass.

\section{Materials and Methods}

\section{Animals and experimental procedures}

Hypophysectomized, male, Sprague-Dawley rats at about $200 \mathrm{~g}$ body mass were obtained from Zivic-Miller. Twenty rats were shipped to NASA Ames Research Center 3 days after hypophysectomy by the parapharyngeal procedure. Following arrival at NASA/Ames Research Center, the rats were stabilized from the trip and adapted to a reversed light cycle in which the cage rooms were illuminated from 8:00 p.m. to 8:00 a.m. All rat care and experimental procedures were performed with red light illumination from 8:00 a.m. to 8:00 p.m. Food and water were provided ad libitum. Animal care and use were in accord with the Ames Research Center Animal Users Guide (AHB 7180). Rats were assigned randomly to one of five groups: i) control, saline injected (CTRL, $\mathrm{n}=4$ ); ii) hindlimb unloaded, salineinjected (UNL, $n=4$ ); iii) hindlimb unloaded, saline-injected plus exercised ( $E X, n=4)$; iv) hindlimb unloaded plus GH-injected (GH, $\mathrm{n}=4)$; v) hind-limb unloaded, GH-injected and exercised $(\mathrm{GH}+\mathrm{EX}, \mathrm{n}=4)$. Prior to hindlimb unloading, rats in the EX and $\mathrm{GH}+\mathrm{EX}$ groups were trained to climb a $1 \mathrm{~m}$ ladder, at 85 degree inclination with a weight that was $70 \%$ of their body mass attached to the base of their tails. Rats in the UNL, EX, GH, and $\mathrm{GH}+\mathrm{EX}$ groups were prepared for hind-limb unloading by cleaning their tails with ethanol and spraying the tails with a benzoin-isopropyl alcohol mixture to protect them from irritation. Approximately two-thirds of the tail was wrapped in a piece of Fast-Trac adhesive tape, covered with a stockinette and secured with fiber tape. The Fast-Trac tape was passed through a wire hook that was suspended from a fishing swivel. The swivel was suspended from an overhead track system. This arrangement allowed the rats to move freely about the cage on their forelimbs. The tail was lifted to a height that prevented contact of the hindlimbs with the floor of the cage. The suspension tracks were blocked such that the rats were unable to touch the sides of the cage with their hindlimbs. ${ }^{22}$ During the 10-day period of hindlimb suspension, the exercise regimen consisted of three daily bouts (8:00 a.m., 12:00
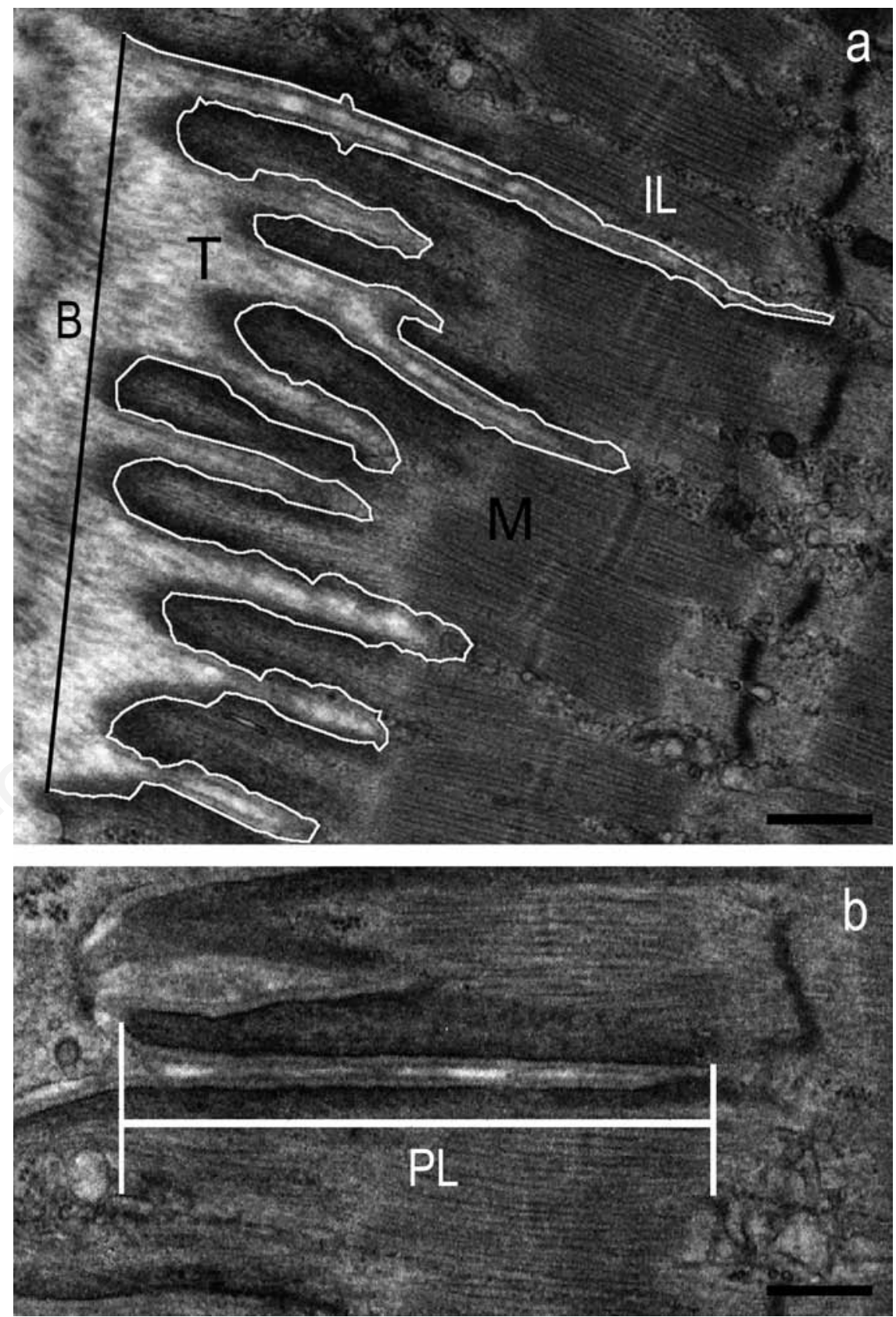

Figure 1. Morphometric analysis of MTJs with ImageJ software. a) Micrograph from a CTRL rat shows a MTJ parallel to the main axis of myofibrils, where the tendon (T) interdigitations penetrate into the muscle $(\mathrm{M})(\mathrm{B}$, base length; IL, interface length). b) Measurement of the primary process extension within the muscle (PL, process length). Scale bars: $0.5 \mu \mathrm{m}$. 
a.m. and 4:00 p.m.) of ten climbs up a ladder while carrying a load equal to $50 \%$ of their body weight attached to their tails. During the same period, the $\mathrm{GH}$ treatment consisted of two daily injections (8:00 a.m. and 4:00 p.m.) of recombinant human GH (a gift from Genentech, South San Francisco, CA, USA), equivalent to a replacement dose in hypophysectomized rats (1 mg/kg body wt, i.p.). ${ }^{23}$

\section{Light and electron microscopy}

The rats were anesthetized deeply with pentobarbital sodium ( $50 \mathrm{mg} / \mathrm{kg}$ body wt, i.p.) and weighed. The plantaris muscle was dissected bilaterally, cleaned of connective tissue and fat, and weighed on a precision scale. Samples were tied to an applicator stick at physiological length and immediately fixed with $1.4 \%$ glutaraldehyde in a $0.2 \mathrm{M}$ sodium cacodylate buffer at $\mathrm{pH} 7.2$ for $1 \mathrm{~h}$ and then minced into small bundles $\left(<1 \mathrm{~mm}^{3}\right)$ of muscle fibers attached to tendon that were fixed in the same solution for an additional hour. After washing, samples were post-fixed with $1 \%$ osmium tetroxide for $1 \mathrm{~h}$ in the same buffer, rinsed in cacodylate buffer and dehydrated in a graded series of ethanols. They were embedded in epoxy resin and sectioned longitudinally. ${ }^{24}$ Semithin sections, stained with $1 \%$ toluidine blue in distilled water, were observed by light microscopy. The tissue blocks then were trimmed and oriented to produce a longitudinal plane of the muscle fibers during thin sectioning, allowing clear MTJ identification. Thin sections were stained with uranyl acetate and lead citrate and analyzed using a Philips CM10 electron microscope (TEM). ${ }^{25,26}$

\section{Morphometric analyses}

Measurements were made only on MTJ processes that were oriented parallel to the longitudinal axis of the myofibrils (Figure 1a). Hundred images of MTJ were evaluated for each group, twenty-five for each rat and twelve or thirteen for each muscle. The base (B) and interface length (IL) of tendon processes at the MTJ were measured in semiautomatic mode, using the image analysis software ImageJ. The IL/B ratio was taken as a measure of MTJ membrane folding and was considered to be an indicator of muscle-tendon interface complexity. ${ }^{6}$ All digitlike processes longer than $0.2 \mu \mathrm{m}$ were considered to be primary processes and the lengths of primary processes (PLs) in each MTJ were measured to determine the extent of muscletendon interpenetration (Figure 1b). The percentages of primary processes that were branched and the number of processes that showed bifurcations also were determined as additional indices of MTJ structural complexity. ${ }^{8}$ All data were expressed as mean values \pm SEM.

\section{Statistical procedures}

Differences in muscle mass, IL/B ratio, primary digit-like process extension, and bifurcation mean among groups were determined using one-way analysis of variance (ANOVA) followed by Tukey HSD post-hoc tests to determine individual group differences. Chi-square tests were used to determine differences in the percentage of branched interdigitations among groups. Significance was set at $\mathrm{P}<0.05$.

\section{Results}

\section{$\mathrm{GH}$ alone and $\mathrm{GH}$ plus exercise} prevented the loss of plantaris mass during chronic unloading

Ten days of unloading resulted in an $~ 8 \%$ decrease in plantaris mass compared to control values (Figure 2). Exercise alone had no effect on muscle mass. In contrast, $\mathrm{GH}$ treatment alone and the combination of $\mathrm{GH}$ and EX treatment increased muscle mass by $\sim 19$ and $25 \%$, respectively, above CTRL values.

\section{Chronic unloading reduced MTJ} structural complexity and disrupted myofibril organization at the junction

The muscles in the CTRL group showed MTJs with interdigitations of the muscle into the tendon and a highly folded MTJ membrane (Figure 3a). The muscle ultrastructure near the MTJ showed a regular sarcomere arrangement with aligned myofilaments and Z-lines (Figure $3 \mathrm{~b}$ ). The cytoplasm of the muscle fibers appeared to be well organized and, at high magnification, undamaged mitochondria and triads were observed in the thin areas between the myofibrils (Figure 3c). Scarce cytosol was evident between the terminal
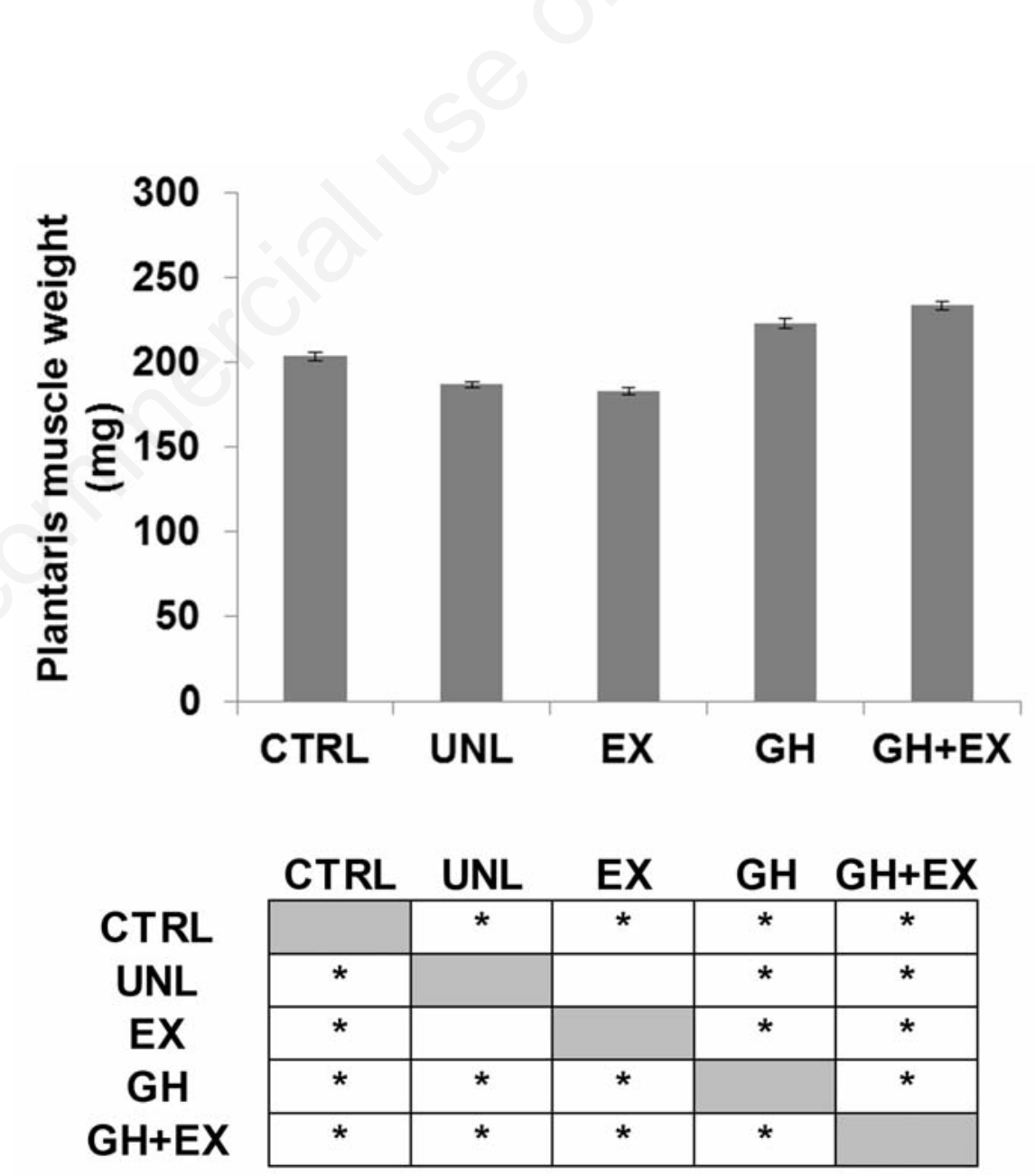

Figure 2. Effects of 10 days of hindlimb unloading (UNL), recombinant human growth hormone treatment $(\mathrm{GH})$, exercise $(\mathrm{EX})$, and the combination of $\mathrm{GH}+\mathrm{EX}$ on plantaris muscle mass $(\mathrm{mg})$. Single factor ANOVA: $\mathrm{F}(4,19)=24.03 \mathrm{P}<\mathbf{0 . 0 1}$; *Tukey HSD P<0.05 CTRL vs UNL, EX, GH and GH+EX; UNL vs GH and GH+EX; EX vs GH and GH+EX; $\mathrm{GH} v s \mathrm{GH}+\mathrm{EX}$. All data were expressed as mean values of plantaris muscle weights of rats for each group \pm SEM. 
myofilaments and the subsarcolemmal dense plaques at the MTJ membrane (Figure 3d).

MTJs in the UNL group showed a reduced complexity compared to those of the CTRL group. Digit-like extensions of the tendon into the muscle were frequently absent and, where present, appeared small and irregular (Figure $3 e$ ). This decrease in structural complexity was reflected by an $\sim 39 \%$ lower IL/B (Figure $4 a)$ and an $\sim 47 \%$ lower PL in the UNL compared to the CTRL group (Figure $4 \mathrm{~b}$ ). No unloading effects, however, were observed in either the percentage of branched processes exceeding $2 \mu \mathrm{m}$ in length (primary processes; Figure 4c) or in the number of bifurcations per primary process (Figure 4d). Thus the reduc- tion of MTJ complexity during unloading was primarily attributable to the lower penetration of digit-like extensions of tendon.

Unloading also affected the organization of myofibrils at the MTJ level. Myofibrils subjacent to the MTJ membrane typically appeared to contain misaligned sarcomeres, with absent or thickened Z-discs (Figure 3e). Unloading
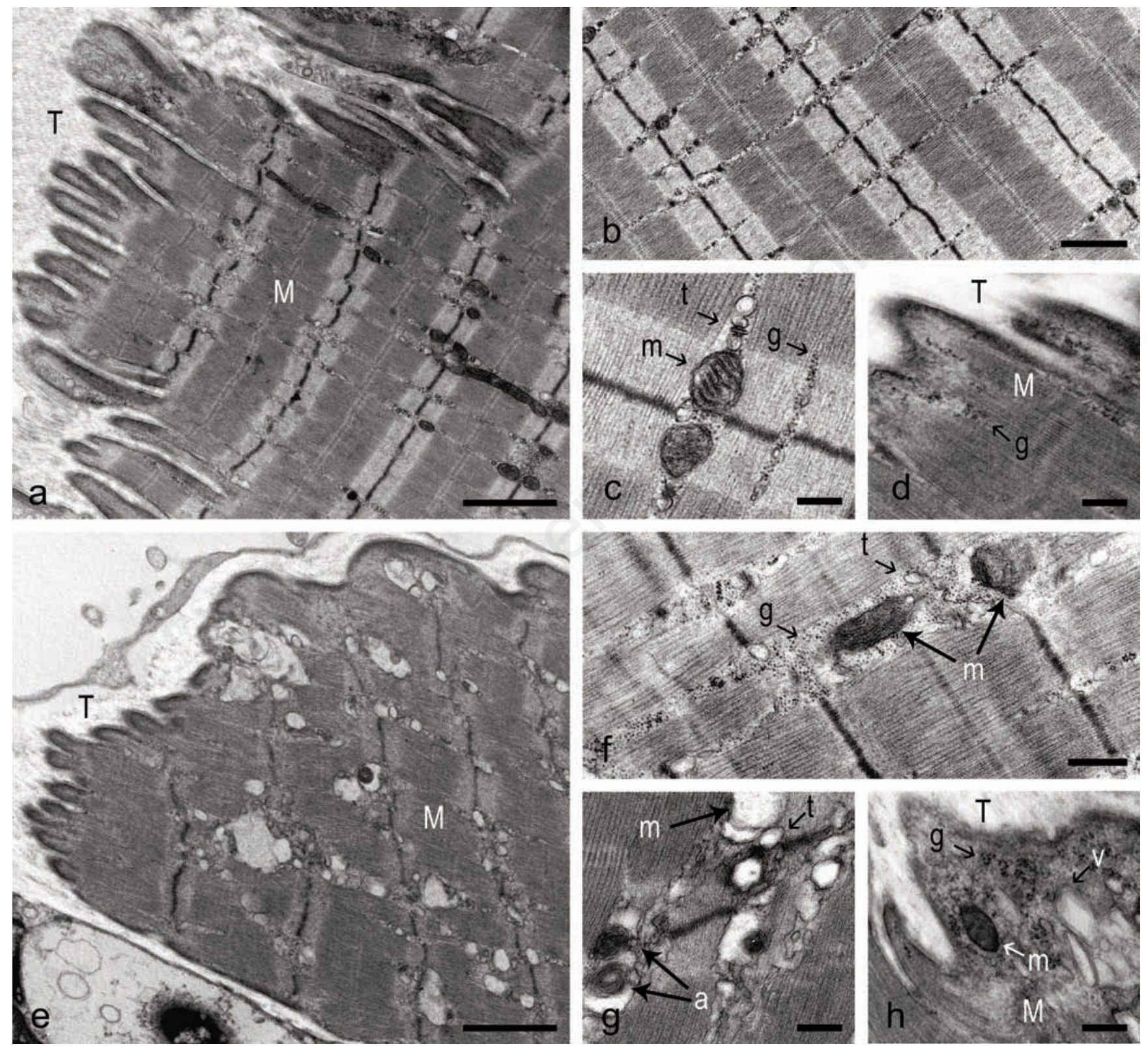

Figure 3. Plantaris MTJ from a CTRL (a-d) and an UNL (e-h) rat. a) Long and several tendon interdigitations penetrate into the muscle mass, parallel to the myofilament orientation. b) Close to MTJ, the muscle ultrastructure appears well organized with aligned Z lines and thin intermyofibrillar cytoplasmic areas. c) The image shows two mitochondria and two triads close to $\mathrm{Z}$ line, one for each side. Moderate glycogen amounts are visible. d) A close contiguity between muscle and tendon appears, showing a small cytoplasmic area and few glycogen particles. e) Short and rare finger-like processes appear. f) Near the MTJ, misaligned sarcomeres are observed. Mitochondria and triads lose their location and the amount of glycogen increases. g) Swollen mitochondria and autophagic vacuoles are evident. h) At the muscle-tendon interface the terminal myofilaments appear separated from extracellular matrix by an increased amount of cytosol, where mitochondria, glycogen granules, and vacuoles appear. M, muscle; T, tendon; m, mitochondria; $t$, triads; $g$, glycogen particles; a, autophagic vacuoles; v, vacuoles. Scale bars: a,e, $2 \mu \mathrm{m}$; b, $1 \mu \mathrm{m}$; f, $0.5 \mu \mathrm{m}$; c,d,g,h, $0.25 \mu \mathrm{m}$. 
also resulted in an increase in the space between myofibrils (Figure 3f) and between myofibrils and the MTJ membrane (Figure $3 \mathrm{~h}$ ) compared to the CTRL group, most likely reflecting the loss of myofibrillar proteins. In addition, there was an increase in the concentration of glycogen granules in the expanded space between myofibrils (Figure $3 \mathrm{f}$ ) and mitochondria often appeared swollen and showed disrupted cristae (Figure 3g), consistent with the shift toward a more glycolytic metabolism in unloaded muscles. ${ }^{27}$

\section{Exercise alone prevented the loss of MTJ membrane folding during unloading, and exercise plus $\mathrm{GH}$ treatment increased the MTJ structural complexity relative to ambulatory controls}

The extent of MTJ folding (IL/B) and the length of digit-like processes of the plantaris MTJs of the rats in the EX group did not differ from CTRL levels (Figure 5a; Figure 4a,b). Unexpectedly, the MTJs in the EX group showed an increased structural complexity compared to those in the CTRL group, i.e., there was an increase in the number of bifurcations per primary process and a strong trend $(\mathrm{P}<0.01)$ for an increase in the percentage of primary digit-like processes (Figure 4c,d).

There were no effects of $\mathrm{GH}$ alone on the structural properties of the MTJs (Figures $4 \mathrm{a}$ $d$ and $5 b$ ). The combination of GH plus EX treatments, however, resulted in an increase in MTJ folding (IL/B) that was greater than with exercise alone (Figures $4 \mathrm{a}$ and $5 \mathrm{c}$ ). In addition, sarcomere organization and alignment as well as intermyofibrillar spacing at the MTJs in the $\mathrm{GH}+\mathrm{EX}$ group were similar to those in the CTRL group. Furthermore, the increased accumulation of glycogen granules between myofibrils observed in the UNL group was not apparent (Figure 5d-f), suggesting that the combined treatment ameliorated the unloading-induced shift toward more glycolytic fibers.

\section{Discussion}

Previous investigations have shown that the structure of MTJs is influenced by changes in muscle loading. The findings of our investigation show that intermittent exercise is sufficient to prevent loss of structural complexity at the MTJ during muscle unloading but that application of $\mathrm{GH}$ to rats experiencing unloading protects muscle mass, without significantly affecting changes in MTJ structure caused by unloading. Nevertheless, the combination of $\mathrm{GH}$ injections with intermittent exercise amplified the protective effects of exercise and provided the best tool for preventing MTJ changes, using the conditions tested in our study. The muscle mass data confirm the numerous works about anabolic effect of $\mathrm{GH}$ on skeletal muscles. ${ }^{28,29}$ The $\mathrm{GH}$, acting either directly or through IGF-1, plays an important role in regulating skeletal muscle growth and development. ${ }^{30}$ It has been shown that chronic GH treatment increases muscle mass and function in GH-deficient patients and animals. ${ }^{31,32}$ These effects are correlated with hypertrophy of existing muscle fibers and proliferation of satellite cells. ${ }^{33}$ Nevertheless, the correlation between muscle mass and its strength is still much debated, during $\mathrm{GH}$ treatment. In fact in older humans, GH therapies have been reported to increase force production ${ }^{34}$ decrease force production ${ }^{35}$ or have no effect. ${ }^{36}$

In GH-treated group, the anabolic effect on
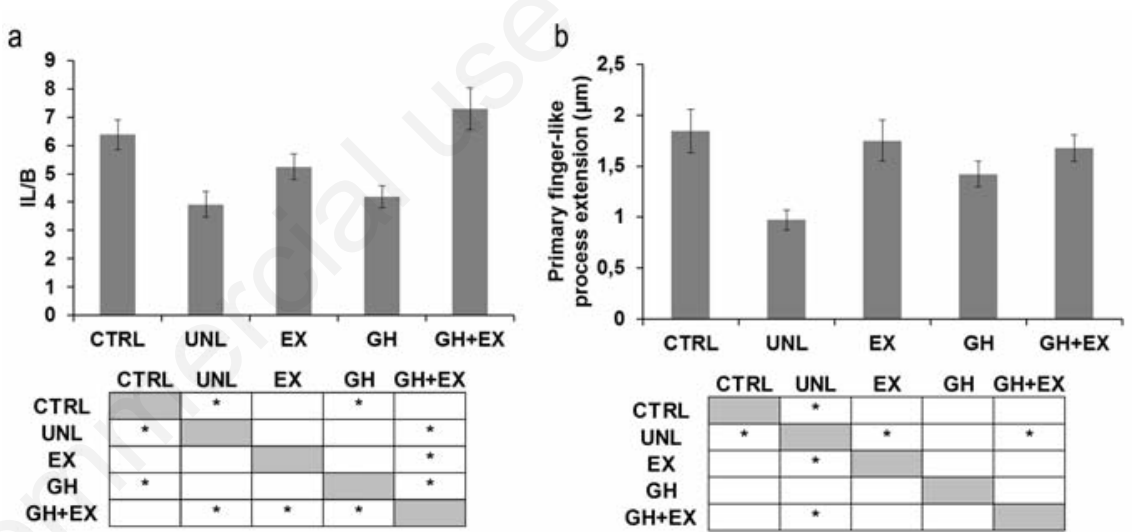

C

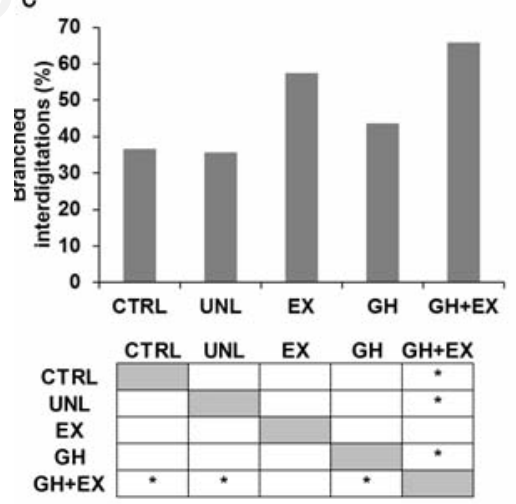

d

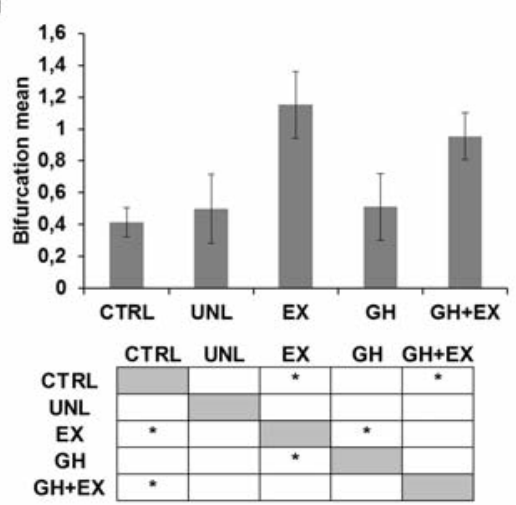

Figure 4. Morphometric analysis of MTJs in each group. a) Interface length/base length ratio (IL/B), Single factor ANOVA: $F(4,102)=7.95 \mathrm{P}<0.01$; *Tukey HSD $\mathrm{P}<0.05 \mathrm{CTRL}$ $v s$ UNL and GH; GH+EX vs UNL, EX and GH. b) Primary finger-like process extension within muscle, Single factor ANOVA: $F(4,101)=4.24 \mathrm{P}<0.01$, *Tukey HSD $\mathrm{P}<0.05$ UNL vs CTRL, EX and GH+EX. c) Percentage of primary finger-like processes longer than 2 um showing bifurcations: $\chi^{2}$-test: ${ }^{*} P<0.05 \mathrm{GH}+\mathrm{EX}$ vs CTRL, UNL and GH. d) Mean of bifurcation of primary finger-like processes longer than $2 \mu \mathrm{m}$, Single factor ANOVA: $\mathrm{F}(4,166)=4,98 \mathrm{P}<0.01$, ${ }^{*}$ Tukey HSD $\mathrm{P}<0.05 \mathrm{CTRL}$ vs $\mathrm{EX}$ and $\mathrm{GH}+\mathrm{EX}$; GH $v$ s EX. All data were expressed as mean values of measurements for each group \pm SEM. 
the neuromuscular junction independent of muscle hypertrophy. ${ }^{40}$ Nerve terminal branching was also affected by exercise training. Neuromuscular junctions from the HIT group showed a greater branching length and number of thinner branches than those of the LIT group. Unexpectedly, these structural modifications appear to be similar to MTJ changes observed in our EX and $\mathrm{EX}+\mathrm{GH}$ groups, where we reveal a growing number of branched interdigitations and a growing number of bifurcations per primary process. Moreover, the same behavior has been described after moderate treadmill running protocols in tibial anterior, EDL and gastrocnemius MTJs of rat. The branching process allows an enlarging of the surface area and, therefore, an increase of tension level; the myotendinous junction can then adjust the shearing force if needed. In addition, it is thought that the meshwork formed by the branching and subsequent joining of the processes is important for regulating tension. ${ }^{8,41}$

From a clinical point of view, our data suggest that the interactive potential between $\mathrm{GH}$ and resistive exercise, in preventing skeletal muscle atrophy should be recognized and that the hormonal treatment alone in GH deficiency could lead to muscle injury related to MTJ adaptation lack. Another interesting observation is that the shift toward a more glycolytic
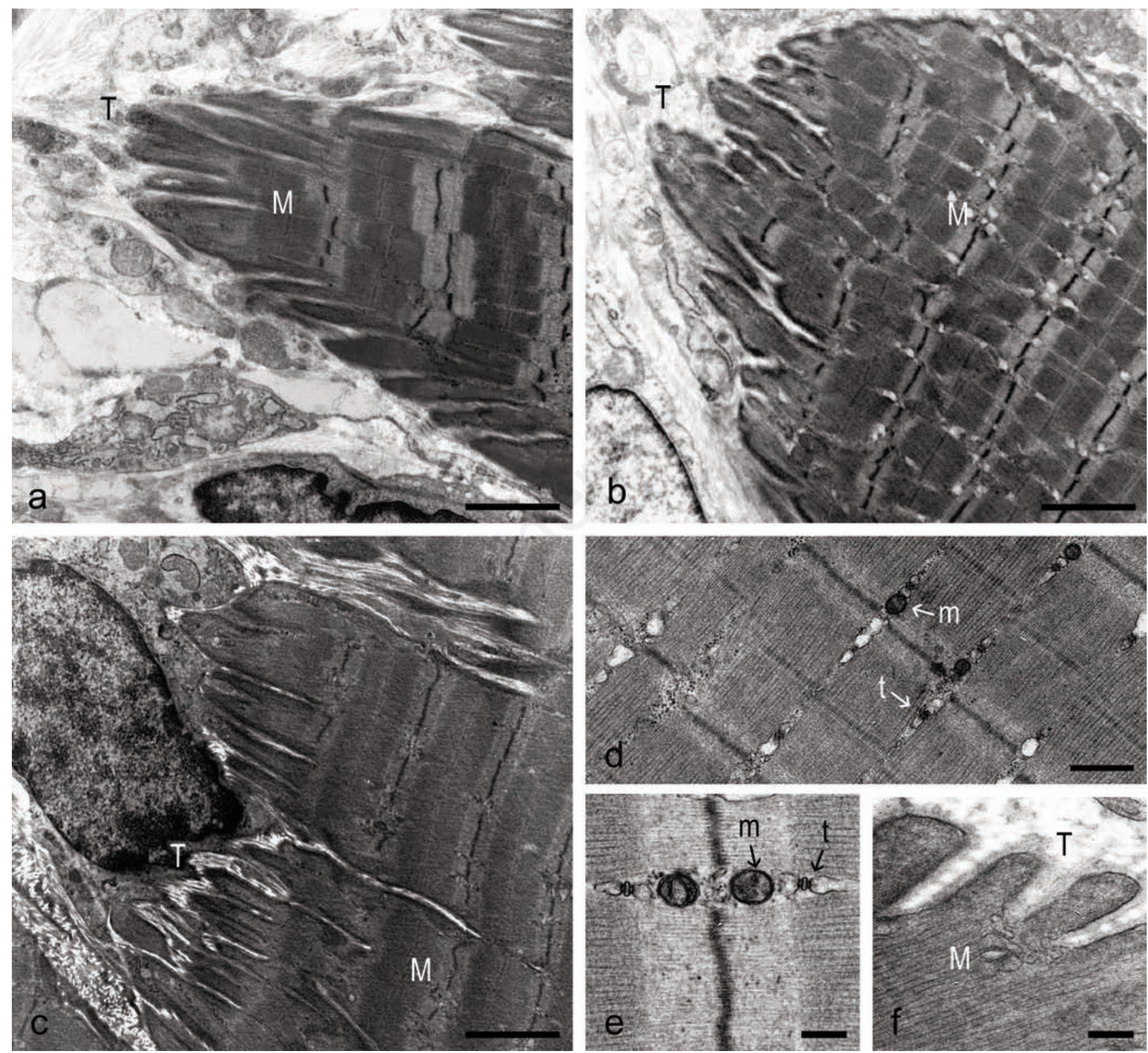

Figure 5. Plantaris MTJ from a rat in the EX (a), GH (b), and GH+EX (c-f) group. a) Tissue condition appears restored and more similar to control compared to the UNL group. The finger-like processes are clearly observable. b) The muscle-tendon interface is similar to UNL group, with rare, irregular and short interdigitations. c) The muscle-tendon interface appears more folded than in the GH and EX groups, presenting several long and branched interdigitations. d) The muscle structure seems to be comparable to that in the CTRL group, with well aligned sarcomeres and regularly distributed myofilaments. e) At high magnification, a normal organization of cytoplasm is evident, with the typical disposition of mitochondria and triads in the thin intermyofibrillar space. $f$ ) Myofilaments appear in close contact with tendon tissue. M: muscle; T: tendon; m: mitochondria; t: triads. Scale bars: a,b,c, $2 \mu \mathrm{m} ; \mathrm{d}, 0.5 \mu \mathrm{m} ; \mathrm{e}, \mathrm{f}, 0.25 \mu \mathrm{m}$. 
metabolism in unloaded muscles, confirmed by numerous works in rodent and human, ${ }^{42,43}$ could be ameliorated by the combined treatment. This finding is in line with the data of Trappe and collaborators, that evidenced how exercise in space may attenuate the fiber shift. In fact postflight muscle samples did not have a large increase in the MHC IIx phenotype and the magnitude of shift away from MHC I fibers was slightly attenuated compared with previous long-duration bed rest studies. ${ }^{44}$

In conclusion, our work demonstrates that reduction of muscle loading affects structure of MTJs of rat plantaris muscle, causing reductions of contact interface between muscle and tendon tissues. In addition, our findings show that intermittent exercise during periods of muscle unloading protect MTJ structure, but GH application alone has no significant protective effect on MTJ architecture. Nevertheless, application of GH in combination with intermittent exercise amplifies the protective effect of exercise.

\section{References}

1. Tidball JG, Lin C. Structural changes at the myogenic cell surface during the formation of myotendinous junctions. Cell Tissue Res 1989;257:77-84.

2. Tidball JG, Quan DM. Modifications in myotendinous junction structure following denervation. Acta Neuropathol 1992;84: 135-40.

3. Russell B, Wenderoth MP, Goldspink PH. Remodeling of myofibrils: subcellular distribution of myosin heavy chain mRNA and protein. Am J Physiol 1992;262:339-45.

4. Tidball JG and Quan DM. Reduction in myotendinous junction surface area of rats subjected to 4-day spaceflight. J Appl Physiol 1992;73:59-64.

5. Zamora AJ, Carnino A, Roffino S, Marini JF. Respective effects of hindlimb suspension, confinement and spaceflight on myotendinous junction ultrastructure. Acta Astronaut 1995;36:693-706.

6. De Palma L, Marinelli M, Pavan M, Bertoni-Freddari C. Involvement of the muscle-tendon junction in skeletal muscle atrophy: an ultrastructural study. Rom J Morphol Embryol 2011;52:105-9.

7. Polican Ciena A, Yokomizo De Almeida SR, De Sousa Bolina C, De Sousa BolinaMatos R, Grassi Rici RE, Pereira Da Silva $\mathrm{MC}$, et al. Ultrastructural features of the myotendinous junction of the sternomastoid muscle in Wistar rats: from newborn to aging. Micron 2012;43:258-62.

8. Curzi D, Salucci S, Marini M, Esposito F, Agnello L, Veicsteinas A, et al. How physi- cal exercise changes rat myotendinous junctions: an ultrastructural study. Eur J Histochem 2012;56:e19.

9. Welser JV, Rooney JE, Cohen NC, Gurpur PB, Singer CA, Evans RA, et al. Myoten dinous junction defects and reduced force transmission in mice that lack alpha7 integrin and utrophin. Am J Pathol 2009;175: 1545-54.

10. Nader GA. Molecular determinants of skeletal muscle mass: getting the "AKT" together. Int J Biochem Cell Biol 2005;37: 1985-96.

11. Russell AP. Molecular regulation of skeletal muscle mass. Clin Exp Pharmacol Physiol 2010;37:378-84.

12. Janssen I, Ross R. Linking age-related changes in skeletal muscle mass and composition with metabolism and disease. $\mathrm{J}$ Nutr Health Aging 2005;9:408-19.

13. Velloso CP. Regulation of muscle mass by growth hormone and IGF-I. Br J Pharmacol 2008;154:557-68.

14. Machida S, Booth FW. Insulin-like growth factor 1 and muscle growth: implication for satellite cell proliferation. Proceedings of the Nutrition Society 2004;63:337-40.

15. Bodine SC, Baar K. Analysis of skeletal muscle hypertrophy in models of increased loading. Methods Mol Biol 2012;798:213-29.

16. Musarò A, Giacinti C, Dobrowolny G, Pelosi L, Rosenthal N. The role of Igf-1 on muscle wasting: a therapeutic approach. Basic Appl Myol 2004;14:29-32.

17. Glass DJ. PI3 kinase regulation of skeletal muscle hypertrophy and atrophy. Curr Top Microbiol Immunol 2010;346:267-78.

18. Jones SW, Hill RJ, Krasney PA, O'Conner B, Peirce N, Greenhaff PL. Disuse atrophy and exercise rehabilitation in humans profoundly affects the expression of genes associated with the regulation of skeletal muscle mass. FASEB J 2004;18:1025-7.

19. Marimuthu K, Murton AJ, Greenhaff PL. Mechanisms regulating muscle mass during disuse atrophy and rehabilitation in humans. J Appl Physiol 2011;110:555-60.

20. Phillips SM. Physiologic and molecular bases of muscle hypertrophy and atrophy: impact of resistance exercise on humanskeletal muscle (protein and exercise dose effects). Appl Physiol Nutr Metab 2009;34:403-10.

21. Linderman JK, Whittall JB, Gosselink KL, Wang TJ, Mukku VR, Booth FW, et al. Stimulation of myofibrillar protein synthesis in hindlimb suspended rats by resistance exercise and growth hormone. Life Sci. 1995;57:755-62.

22. Grindeland RE, Roy RR, Edgerton VR, Grossman EJ, Mukku VR, Jiang B, et al. Interactive effects of growth hormone and exercise on muscle mass in suspended rats. Am J Physiol 1994;267:316-22.

23. Linderman JK, Gosselink KL, Booth FW, Mukku VR, Grindeland RE. Resistance exercise and growth hormone as countermeasures for skeletal muscle atrophy in hindlimb-suspended rats. Am J Physiol 1994;267:365-71.

24. Law DJ, Caputo A, Tidball JG. Site and mechanics of failure in normal and dystrophin-deficient skeletal muscle. Muscle Nerve 1995;18:216-23.

25. Salucci S, Battistelli M, Burattini S, Squillace C, Canonico B, Gobbi P, et al. C2C12 myoblast sensitivity to different apoptotic chemical triggers. Micron 2010; 41:966-973.

26. Luchetti F, Canonico B, Curci R, Battistelli M, Mannello F, Papa S, Tarzia G, Falcieri E. Melatonin prevents apoptosis induced by UV-B treatment in U937 cell line. J Pineal Res 2006;40:158-167.

27. Fitts RH, Riley DR, Widrick JJ. Functional and structural adaptations of skeletal muscle to microgravity. J Exp Biol 2001;204: 3201-8.

28. Cartee GD, Bohn EE, Gibson BT, Farrar RP. Growth hormone supplementation increases skeletal muscle mass of old male Fischer 344/brown Norway rats. J Gerontol A Biol Sci Med Sci. 1996;51:B214-9.

29. Simon D, Alberti C, Alison M, Lehenaff L, Chevenne D, Boizeau P, et al. Effects of recombinant human growth hormone for 1 year on body composition and muscle strength in children on long-term steroid therapy: randomized controlled, delayedstart study. J Clin Endocrinol Metab 2013; 98:2746-54.

30. Florini JR, Ewton DZ, Coolican SA. Growth hormone and the insulin-like growth factor system in myogenesis. Endocr Rev 1996;17:481-517.

31. Gibney J, Wallace JD, Spinks T, Schnorr L, Ranicar A, Cuneo RC, et al. The effects of 10 years of recombinant human growth hormone $(\mathrm{GH})$ in adult GH-deficient patients. J Clin Endocrinol Metab 1999;84: 2596-602.

32. Roy RR, Tri C, Grossman EJ, Talmadge RJ, Grindeland RE, Mukku VR, et al. IGF-I, growth hormone, and/or exercise effects on non-weight-bearing soleus of hypophysectomized rats. J Appl Physiol 1996;81: 302-11.

33. Kim H, Barton E, Muja N, Yakar S, Pennisi $P$, Leroith D. Intact insulin and insulin-like growth factor-I receptor signaling is required for growth hormone effects on skeletal muscle growth and function in vivo. Endocrinology 2005;146:1772-9.

34. Welle S, Thornton C, Statt M, McHenry B. Growth hormone increases muscle mass and strength but does not rejuvenate 
myofibrillar protein synthesis in healthy subjects over 60 years old. J Clin Endocrinol Metab 1996;81:3239-43.

35. Zachwieja JJ, Toffolo G, Cobelli C, Bier DM, Yarasheski KE. Resistance exercise and growth hormone administration in older men: effects on insulin sensitivity and secretion during a stable-label intravenous glucose tolerance test. Metabolism 1996;45:254-60.

36. Papadakis MA, Grady D, Black D, Tierney MJ, Gooding GA, Schambelan M, et al. Growth hormone replacement in healthy older men improves body composition but not functional ability. Ann Intern Med 1996;124:708-16.

37. Chihara K, Fujieda K, Shimatsu A, Miki T, Tachibana K. Dose-dependent changes in body composition during growth hormone (GH) treatment in Japanese patients with adult $\mathrm{GH}$ deficiency: a randomized, place- bo-controlled trial. Growth Horm IGF Res 2010;20:205-11.

38. Kaspers S, Ranke MB, Han D, Loftus J, Wollmann H, Lindberg A, et al. Impli cations of a data-driven approach to treatment with growth hormone in children with growth hormone deficiency and Turner syndrome. Appl Health Econ Health Policy 2013;11:237-49.

39. Meazza C, Pagani S, Pietra B, Tinelli C, Calcaterra V, Bozzola E, et al. Different long-term response to growth hormone therapy in small- versus appropriate-forgestational-age children with growth hormone deficiency. Horm Res Paediatr 2013; 25:214-9.

40. Deschenes MR, Maresh CM, Crivello JF, Armstrong LE, Kraemer WJ, et al. The effects of exercise training of different intensities on neuromuscular junction morphology. J Neurocytol 1993;22:603-15.
41. Kojima H, Sakuma E, Mabuchi Y, Mizutani J, Horiuchi 0, Wada I, et al. Ultrastructural changes at the myotendinous junction induced by exercise. J Orthop Sci 2008;13: 233-9.

42. Talmadge RJ, Roy RR, Edgerton VR. Distribution of myosin heavy chain isoforms in non-weight-bearing rat soleus muscle fibers. J Appl Physiol 1996;81: 2540-6.

43. Zhou MY, Klitgaard H, Saltin B, Roy RR, Edgerton VR, Gollnick PD. Myosin heavy chain isoforms of human muscle after short-term spaceflight. J Appl Physiol 1995;78:1740-4.

44. Trappe S, Costill D, Gallagher P, Creer A, Peters JR, Evans H, Riley DA, Fitts RH. Exercise in space: human skeletal muscle after 6 months aboard the International Space Station. J Appl Physiol 2009;106: 1159-68. 